\title{
A Review Study on Mathematical Methods for Fault Detection Problems in Induction Motors
}

\author{
E. Ayaz
}

\begin{abstract}
Induction motors are frequently used in industrial processes. Failure of these machines may cause economic, quality and safety losses. In this paper, the mathematical methods used in detection of mechanical and electrical faults of these motors are reviewed together with theory and application examples on the current and vibration data which is acquired during performance tests of the motors followed by accelerated aging.
\end{abstract}

Index Terms-Induction motor, fault detection, aging, signal processing

\section{INTRODUCTION}

$\mathrm{I}_{\mathrm{i}}$ NDUCTION motors are widely used electrical drives in industrial processes due to their wide power range, simple and rugged structure. When the motor ages or a fault occurs in the motors, these may cause losses in their efficiency, economic and safe operation of industrial processes. The faults can be categorized into as being mechanical or electrical faults. According to the statistical surveys performed on motors revealed that $41 \%$ of failures are resulted from bearing faults, $37 \%$ of them are from stator faults, $10 \%$ is from rotor faults, and $12 \%$ are from other faults such as unbalanced phase supply, soft foot, asymmetries in the magnetic circuits etc. ${ }^{(1-7)}$

This paper addresses fault detection methods in induction motors together with theory and applications on experimental data acquired during performance test of the motors subjected to accelerated aging ${ }^{(6-7)}$. Detection of eccentricity fault ${ }^{(7-9)}$, bearing fault ${ }^{(10-18)}$, and stator insulation fault ${ }^{(19-20)}$ is considered. Applications of statistical methods, power spectral density analysis, coherence analysis, continuous and discrete wavelet transform, autoregressive modeling method, adaptive neuro-fuzzy inference system, artificial neural network is presented by means of the experimental data.

\section{MATHEMATICAL METHODS}

This section includes mathematical methods used in signal analysis for fault detection and diagnosis studies. These are frequency domain methods, time-frequency/scale domain methods, stochastic methods, and soft computing methods.

\subsection{FREQUENCY DOMAIN METHODS}

Since different fault types generate different frequency spectrum distributions, the diagnosis results are based on frequency features of signals. Frequency content of a signal at frequency $m \Delta f$ can be found by Fourier transform given as below ${ }^{(21-22)}$

$$
X(m \Delta f)=\sum_{k=0}^{N-1} x(k \Delta t) \exp \left(-\frac{j 2 \pi k m}{N}\right)
$$

where $N$ is the number of samples, $\Delta f$ is the frequency resolution, $m$ is integer number and $\Delta t$ is the data-sampling interval. The auto-power spectral density (APSD) of $x(t)$ is estimated as

$$
S_{x x}(f)=\frac{1}{N}|X(m \Delta f)|^{2}, \quad f=m \Delta f
$$

The cross power spectral density (CPSD) between $x(t)$ and $y(t)$ is similarly estimated. The statistical accuracy of the estimate in Equation (2) increases as the number of data points or the number of blocks of data increases.

The cause and effect relationship between two signals or the commonality between them is generally estimated using the coherence function. The coherence function is given by

$$
\gamma_{x y}(f)=\frac{\left|S_{x y}(f)\right|}{\sqrt{S_{x x}(f) S_{y y}(f)}}, \quad 0<\gamma_{x y}<1
$$

where $S_{x x}$ and $S_{y y}$ are the APSD's of $x(t)$ and $y(t)$, respectively, and $S_{x y}$ is the CPSD between $x(t)$ and $y(t)$. A value of coherence close to unity indicates highly linear and close relationship between the two signals.

\subsection{TIME-FREQUENCY/SCALE DOMAIN METHODS}

To find time localization of the frequency content of the signal short-time Fourier transform (STFT) can be used which is defined as ${ }^{(23-24)}$

$$
\operatorname{STFT}(\tau, f)=\int_{-\infty}^{\infty} x(t) g(t-\tau) \exp [-2 \pi f t] d t
$$


In STFT, the signal $x(t)$ is first windowed using different type of window functions $g(t)$ such as triangular window, rectangular window, Gaussian window etc. centered at time location $\tau$. Then Fourier Transform of resulting windowed signal is taken. This gives the STFT of signal for particular time. As window slides along time axis, so basically STFT maps input signal $x(t)$ into two dimensional function in a timefrequency plane with fixed resolution.

Wavelet transform can be used to separate the signal into frequency bands and to get variable resolution which is good time resolution for rapidly changing signals and good frequency resolution for slowly changing signals. The continuous wavelet transform is defined as

$$
W_{f}(a, b)=\int_{-\infty}^{\infty} x(t) \psi_{a, b}(t) d t
$$

where

$$
\psi_{a, b}(t)=\frac{1}{\sqrt{|a|}} \psi\left(\frac{t-b}{a}\right) ; \quad a, b \in R ; a \neq 0
$$

$\psi$ is called the mother wavelet. The dilation parameter $a$ controls the scale or frequency of wavelet, and the translation parameter $b$ controls the position of the wavelet in time. The parameters $a$ and $b$ are defined as $a=a_{0}^{j}, b=n b_{0} a_{0}^{j}$ where $n, j \in Z, a_{0}>1$, and $b_{0}>0$, the Discrete Wavelet Transformation (DWT) is given as

$$
D W T[j, k]=\frac{1}{\sqrt{a_{0}^{j}}} \sum_{n} x[n] \psi\left[\frac{k-n b_{0} a_{0}^{j}}{a_{0}^{j}}\right]
$$

S. Mallat introduced an efficient algorithm to perform the DWT known as the Multi-Resolution Analysis (MRA). The MRA is similar to a two-channel sub-band coder used in highpass and low-pass filters, from which the original signal can be reconstructed.

The frequency decomposition of the signal is shown schematically (Fig.1). The low-frequency sub-band is referred to as approximation $a_{i}$ and the high-frequency sub-band by detail $d_{i}$. Thus, at the second stage the signal may be reconstructed as

$$
S=a_{2}+d_{1}+d_{2}
$$

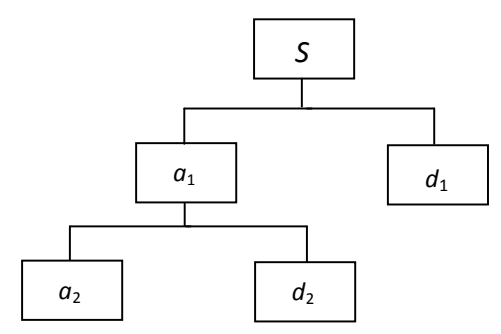

Fig.1. Signal decomposition at the second stage.

\subsection{STOCHASTIC METHODS}

This section gives statistical methods and time series modeling methods.

\subsection{STATISTICAL METHODS}

Several statistical parameters, calculated in the time domain, are generally used to define average properties of machinery data. The two basic parameters are the mean value $\mu$ and the standard deviation $\sigma$. For a given data set $\left\{x_{i}\right\}$ these are defined as follows ${ }^{(25)}$ :

$$
\begin{gathered}
\mu=\frac{1}{N} \sum_{i=1}^{N} x_{i} \\
\sigma=\sqrt{\frac{1}{N} \sum_{i=1}^{N}\left(x_{i}-\mu\right)^{2}}
\end{gathered}
$$

where $N$ is the number of the data points. For the Gaussian (normal) probability distribution, two parameters that reflect the departure from the normal distribution are skewness $(c)$ and kurtosis $(k)$. These are calculated as follows.

$$
\begin{gathered}
c=\frac{\left[\frac{1}{N} \sum_{i=1}^{N}\left(x_{i}-\mu\right)^{3}\right]}{\sigma^{3}} \\
k=\frac{\left[\frac{1}{N} \sum_{i=1}^{N}\left(x_{i}-\mu\right)^{4}\right]}{\sigma^{4}}
\end{gathered}
$$

For a perfect normal distribution, $c$ is equal to zero. A negative value is due to skewness towards lower values while a positive value indicates non-symmetry towards higher values. For small data sets, one often gets values that differ from zero. The kurtosis or flatness $k$, is very close to unity for a normal distribution. These statistical parameters may be used to perform a quick check of the changes in the statistical behaviour of a signal.

\subsection{TIME SERIES MODELING METHODS}

In time series analysis the autoregressive (AR) modeling method is used commonly due its simplicity and ability to show sharp peaks in the frequency domain. The AR method establish the mathematical model for regression and forecast, hence the AR coefficients of the model represent the signal features, and can be used for fault detection purposes ${ }^{(16,26)}$.

AR modeling is a parametric method and represents the signal as a linear combination of its previous values plus an error term. AR model of order $p$ for the signal $x(n)$ is given as

$$
x(n)+\sum_{k=1}^{p} a_{k} x(n-k)=v(n)
$$


where $a_{k}$ for $k=0,1, \ldots, p$ are AR coefficients and $v(n)$ is the white noise with variance $\sigma_{v}^{2}$. The filter coefficients are determined using the autocorrelation sequence of the AR process which satisfies the Yule-Walker equations given by

$$
r_{x}(k)+\sum_{l=1}^{p} a_{l} r_{x}(k-l)=\sigma_{v}^{2} \delta(k) ; k \geq 0
$$

where $\delta(k)$ is the unit sample sequence. Thus given the autocorrelations $r_{x}(k)$ for $k=0,1, \ldots, p$ these equations can be solved for the AR coefficients $a_{k}$ and $\sigma_{v}$. In most applications $r_{x}(k)$ is unknown and estimated from a sample realization of the process. Given $x(n)$ for $0 \leq n \leq N$, where $N$ is the number of samples, $r_{x}(k)$ is estimated using the sample autocorrelation

$$
\hat{r}_{x}(k)=\frac{1}{N} \sum_{n=0}^{N-1} x(n) x(n-k)
$$

Order selection is important in terms of to get less error between signal and its AR model. And also there must be enough number of coefficients, but not too much, to diagnose faults conveniently. Akaike's Information Criteria (AIC) can be used for order selection and given as follows

$$
\operatorname{AIC}(p)=N \ln \left(\sigma_{v}^{2}\right)+2 p
$$

\subsection{SOFT COMPUTING METHODS}

The relation matrix between fault types and fault features extracted from vibration, current, temperature signals can be too complicated to determine fault types by this matrix with human inference. Intelligent methods like artificial neural networks (ANN) and adaptive neuro-fuzzy inference system (ANFIS) can be used for classification and pattern recognition.

\subsubsection{ARTIFICIAL NEURAL NETWORKS}

ANN is an interconnection of computational elements known as neurons ${ }^{(27)}$. Each neuron has multi inputs from other neurons with assigned weights. The output of a neuron is computed by summing of all the weighted inputs and then passing it through a function. ANN consists of one or more layers of neurons in interconnected topology (Fig.2).

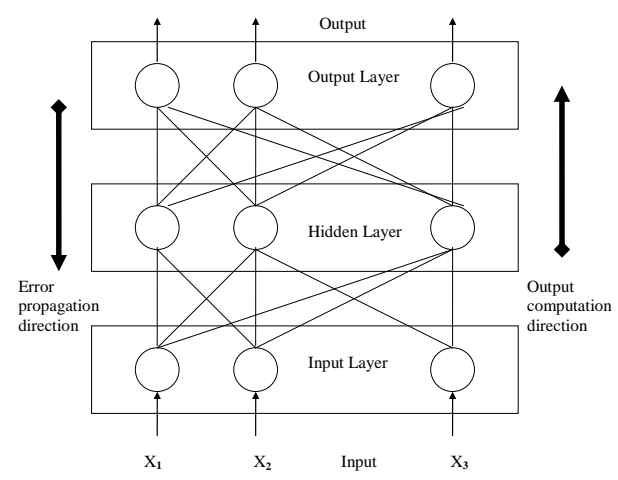

Fig.2. Topology of a feed forward neural network with three layers.
The backpropagation learning algorithm is widely used to determine input-output relationship of a system. It is an iterative gradient search algorithm which adjust each weight in a multilayer network so as to reduce the error in the outputs. It works by propagating errors backward from the output layer. For a three layer ANN with $I$ inputs, one hidden layer with $J$ neurons and $K$ outputs neurons, the error function is

$$
E=\frac{1}{2} \sum_{p=1}^{P} \sum_{k=1}^{K}\left(y_{k p}-d_{k p}\right)^{2}
$$

where $y_{k p}$ and $d_{k p}$ are the actual and desired outputs of the pattern respectively. Individual weight adjustment between the hidden layer and the output layer for pattern $p$ are computed by

$$
\Delta \omega_{k j}=-\eta \frac{\partial E_{p}}{\partial \omega_{k j}}
$$

where $j=1,2, \ldots J$ and $\eta$ is a constant learning rate. The weights between hidden and output layers are adjusted using the recursive formula given as

$$
\omega_{k j}(n+1)=\omega_{k j}(n)+\eta \Delta \omega_{k j}
$$

where $n$ is the iteration number. Similar weight adjustment formulas can be obtained between the input and output layers by changing the indices from $k$ to $i$.

\subsubsection{ADAPTIVE NEURO-FUZZY INFERENCE SYSTEM}

Adaptive neuro-fuzzy inference system (ANFIS) is an implementation of a fuzzy logic inference system with the architecture of a five-layer feed-forward network $(18,19,27,28)$. With this way ANFIS uses the advantages of learning capability of neural networks and inference mechanism similar to human brain provided by fuzzy logic. The architecture of ANFIS with two inputs, one output and two rules is given (Fig.3). Here $x, y$ are inputs, $f$ is output, the circles represent fixed node functions and squares represent adaptive node functions. This is a Sugeno-type fuzzy system, where the fuzzy IF-THEN rules have the following form:

Rule 1: If $x$ is $A_{1}$ and $y$ is $B_{1}$ then $f_{1}=p_{1} x+q_{1} y+r_{1}$ Rule 2: If $x$ is $A_{2}$ and $y$ is $B_{2}$ then $f_{2}=p_{2} x+q_{2} y+r_{2}$

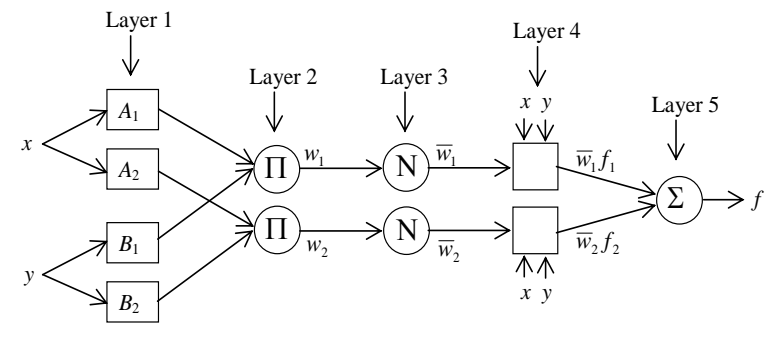

Fig.3. ANFIS architecture with two rules.

The operation of each layer is as follows: 
Layer 1 called fuzzification layer and it fuzzify the values of the input variables according to a membership function. This layer forms the antecedents of the fuzzy rules (IF part). Generalized bell membership function (Fig.4) is popular for specifying fuzzy sets because of their smoothness and concise notation. The parameters $\left\{a_{i}, b_{i}, c_{i}\right\}$ of the membership function are called as premise parameters where $i$ denotes the node number.

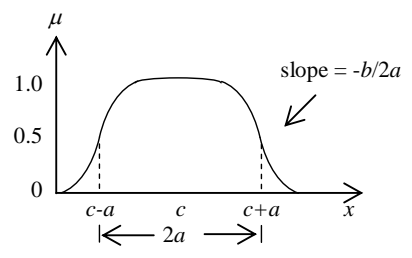

Fig.4. Generalized bell function.

Layer 2 is rules layer and performs fuzzy AND operation at the nodes. Thus the output is the product of all incoming signals and represents the firing strength of each rule. Layer 3 is normalization layer. Layer 4 is fuzzy inference or consequent layer which estimates the rule's output (THEN part). The parameters $\left\{p_{i}, q_{i}, r_{i}\right\}$ of each rule's output are referred as consequent parameters. Layer 5 is called defuzzification layer and calculates the sum of the outputs of all the rules. Learning of ANFIS is done using hybrid learning procedure given in Table 1. This algorithm combines backpropagation gradient descent and least squares method for identification of premise and consequent parameters in such a way that ANFIS output matches the training data.

Table 1. Hybrid learning procedure.

\begin{tabular}{|l|l|l|}
\hline & Forward pass & Backward pass \\
\hline Premise parameters & Fixed & Gradient descent \\
\hline Consequent parameters & Least squares & Fixed \\
\hline Signals & Node outputs & Error signals \\
\hline
\end{tabular}

\section{CASE STUDIES}

In this section example applications of mathematical methods on experimental data for detection of mechanical and electrical faults in induction motors are given. Detection of eccentricity fault, bearing fault, and insulation fault is considered. For this purpose $5 \mathrm{HP}$, three phases, four poles induction motor is subjected to bearing damage and winding insulation damage as well as thermal and chemical aging ${ }^{(6-7)}$. After each aging cycle the data is collected at $12 \mathrm{kHz}$ sampling frequency under $100 \%$ load condition. Seven aging cycle is performed until the motor is broken down.

\subsection{MECHANICAL FAULTS AND THEIR DETECTION}

Airgap eccentricity (Non-uniform air gap):

Air gap eccentricity may be the consequence of bearing, wear or bearing failure, bad motor assembly with rotor unbalance or a rotor which is not perfectly centered ${ }^{(4,8-9)}$. This eccentricity causes anomalies in the air gap flux density and creates frequency side bands at around the supply frequency of the stator current signal for each phase. The side-band frequencies caused by the dynamic eccentricity are given as

$$
\begin{gathered}
f_{e c c}=f_{e}\left[1 \mp k\left(\frac{1-s}{p / 2}\right)\right]=\left|f_{e} \mp k f_{r}\right|, k \\
=1,2,3, \ldots
\end{gathered}
$$

where $f_{e}$ is the electrical supply frequency, $s$ is the per unit slip, $p$ is the number of poles, and $f_{r}$ is the rotor speed in Hz. Slip $s$ $=\left(f_{s}-f_{r}\right) / f_{s}$, where $f_{s}$ is the synchronous frequency.

The side-band effects of the motor current signals both of the healthy and faulty cases by means of their power spectral density variations at around supply frequency $60 \mathrm{~Hz}$ is shown in (Fig.5a) and (Fig.5b).

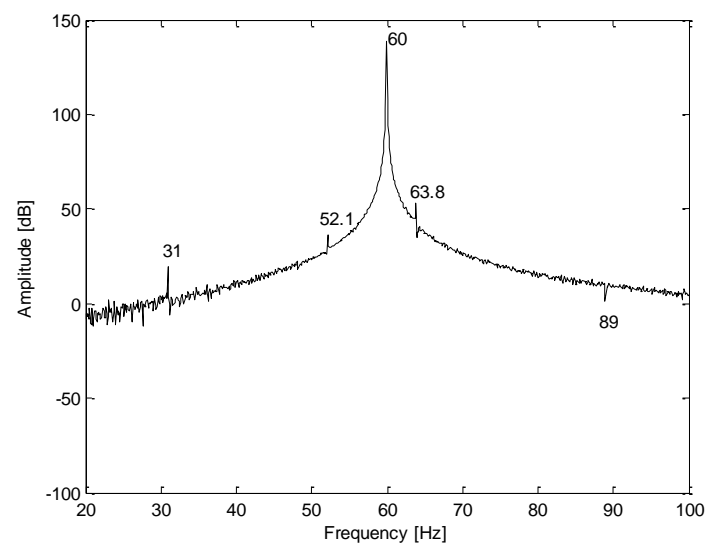

(a) APSD for healthy case.

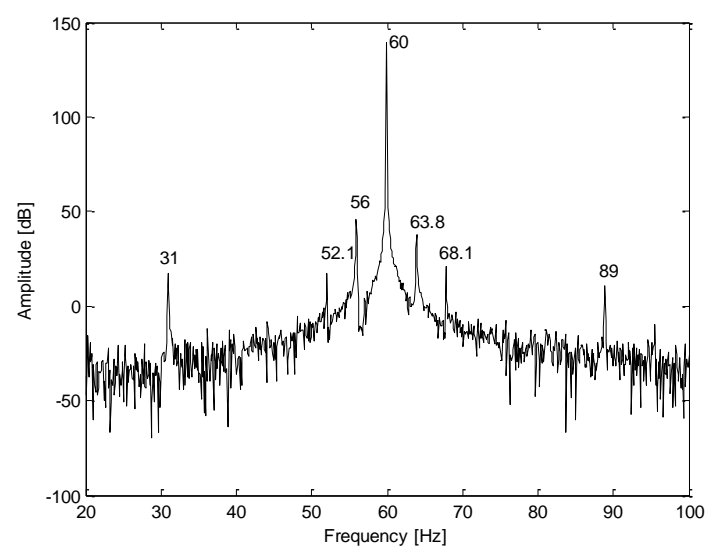

(b) APSD for aged case.

Fig.5. APSD variations of current signals

The big amplitudes defined as side-band frequencies (Fig.5b), which are appeared at around the fundamental frequency $60 \mathrm{~Hz}$ with difference of $4 \mathrm{~Hz}$, indicate the rotor eccentricity. Here the side-band frequencies $\left(f_{s b}\right)$ can be defined by the following equation

$$
f_{s b}=f_{e}(1 \mp 2 k s)
$$

where $k$ is an integer. In this application $f_{r}=1800 / 60=30 \mathrm{~Hz}$ and $f_{r}=1742 / 60=29.03 \mathrm{~Hz}$. Taking $k=1,2$ and $s=0.032$, from the Eq. (21), all side-band frequency values can be found at 52, 56, 64 and $68 \mathrm{~Hz}$ (Fig.5b). Also, comparing the Fig.5a and Fig.5b the 
other important frequency components can be determined at 31 $\mathrm{Hz}$ and $89 \mathrm{~Hz}$. These are the rotor eccentricity frequencies and they can be defined between the rotational frequency $\left(f_{r}\right)$ and fundamental frequency $\left(f_{e}\right)$ by Equation (20).

\section{Bearing faults:}

One cause of premature bearing failure is the passage of electrical current through the bearing and it is known as electrical discharge machining or fluting ${ }^{(1-3)}$. A severe damage is a source of audible bearing noise. The surface degradation of bearing races and rolling elements results in extreme vibration levels and eventual bearing failure.

Statistical properties of vibration signal can be investigated for detecting the fault ${ }^{(10-11)}$. The deviation between statistical parameters for the healthy and the faulty cases are compared as given in Table 2 . The healthy and faulty vibration signals in time domain and their amplitude probability density functions (histograms) is shown in (Fig.6). These results indicate that the signals have a normal distribution and the overall standard deviation has increased by a factor of about 6 , showing the damage.

Table 2. Statistical parameters of vibration signal

\begin{tabular}{|l|l|l|}
\hline & Healthy & Faulty \\
\hline Mean & 0.0016 & 0.0030 \\
\hline Standard Deviation & 0.1135 & 0.6040 \\
\hline Skewness & 0.0591 & -0.0060 \\
\hline Kurtosis & 2.9266 & 3.0093 \\
\hline
\end{tabular}
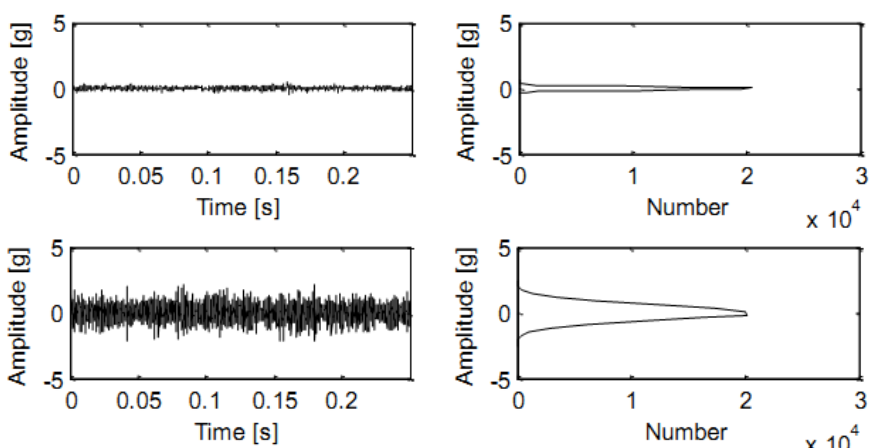

Fig.6. Accelerometer signal waveforms: (a) Baseline. (b) Final aged cycle. (c) Probability density functions of a) and b).

Bearing faults can be diagnosed by frequency domain analysis of vibration signals ${ }^{(12-14)}$. When a fault occurs in any bearing components, this creates vibrations at characteristic frequencies defined by the bearing geometry. These characteristic fault frequencies can be calculated by using either the bearing geometry or approximate formulas for the bearings which have the number of balls between six and twelve as below:

Cage Frequency: $f_{c}=0.4 f_{r}$

Ball Pass Frequency of the Outer Race (BPFO): $f_{o}=0.4 N_{b} f_{r}$

Ball Pass Frequency of the Inner Race (BPFI): $f_{i}=0.6 N_{b} f_{r}$

Ball Spin Frequency $\left(f_{b}\right)$ is calculated using the bearing data provided by the bearing manufacturer. Here $f_{r}$ is the rotor frequency and $N_{b}$ is the number of balls. In this study, the motor speed is $1742 \mathrm{~min}^{-1}$ and then the rotor frequency is calculated as $f_{r}=1742 / 60=29.03 \mathrm{~Hz}$. The number of balls in the bearing is $N_{b}=9$. The characteristic frequencies are calculated as $f_{c}=$ $11.6 \mathrm{~Hz}, f_{o}=104.5 \mathrm{~Hz}$, and $f_{i}=156.7 \mathrm{~Hz}$. Ball Spin Frequency is calculated as $f_{b}=136.9 \mathrm{~Hz}$ for the given rotational speed.

The characteristic bearing frequencies and its harmonics occur when the bearing has a fault. The variations that are produced by the air gap eccentricity due to bearing defect generate stator currents that are related to these characteristic bearing frequencies $\left(f_{v}\right)$

$$
f_{\text {bng }}=\left|f_{e} \mp m f_{v}\right|, \quad m=1,2,3, \ldots
$$

where $f_{v}$ is one of the characteristic bearing frequencies namely $f_{c}, f_{o}, f_{i}, f_{b}$.

The characteristic bearing frequencies are remarkable when the bearings have single point defects. If the defects are scattered on the bearing components, an increase is seen in the high frequency region of the vibration spectrum. From the power spectral density (Fig.7) of vibration signals for the healthy and faulty case, it is clear that there is an increase in the signal energy for the frequency ranges $1.5-4 \mathrm{kHz}$ are due to frosting on the surface of bearing elements, caused by material removal by pitting. Data acquisition system has a low-pass filter with the cutoff frequency at $4 \mathrm{kHz}$.

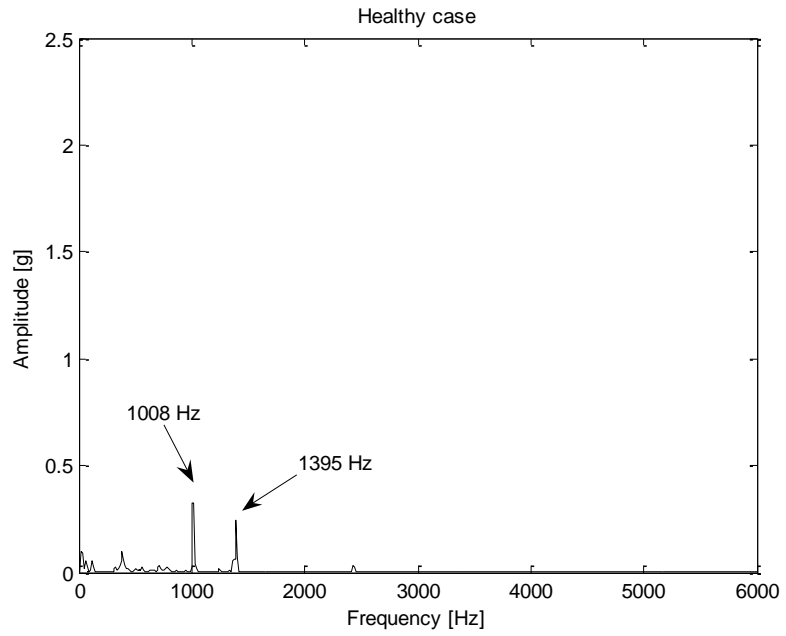

(a)

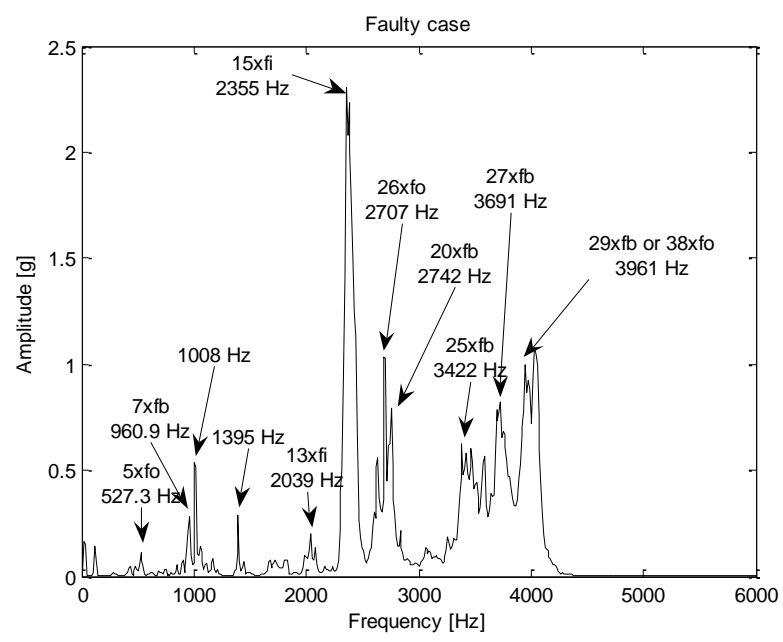

(b) 
Fig.7. Power spectral densities of vibration signals (a) healthy, (b) aged cases.

The coherence function (Fig.8) indicates that the most dominant frequency values, where motor current and vibration signals are correlated, are located at $234 \mathrm{~Hz}$ and $469 \mathrm{~Hz}^{(8-9)}$. The side-band frequency due to the dynamic eccentricity is

$$
f_{e c c}=60+6(29.03)=234 \mathrm{~Hz}
$$

The gap eccentricity generated current due to the bearing defect is

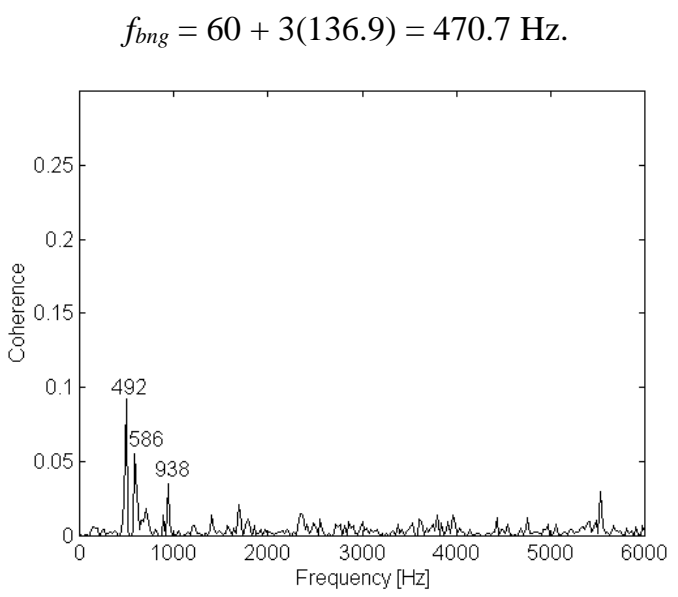

(a)

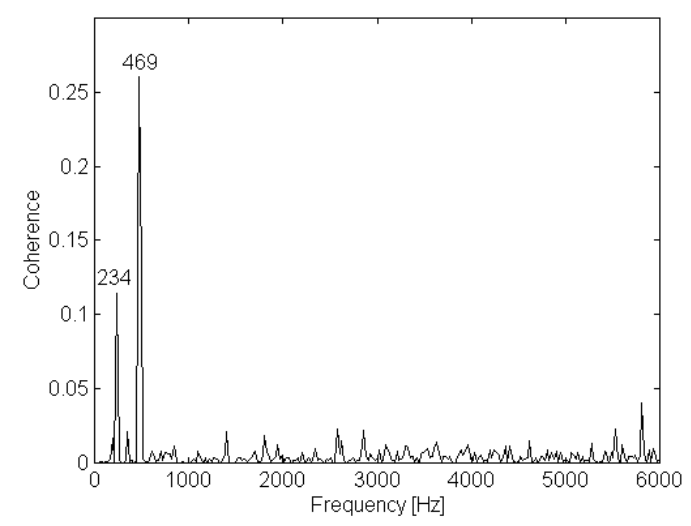

(b)

Fig.8. The coherences for healthy (a) and faulty (b) cases.

The coherence function between the motor current and accelerometer signals at the $234 \mathrm{~Hz}$ and $470.7 \mathrm{~Hz}$ has increased by a factor of 2.5 in the $200-500 \mathrm{~Hz}$ frequency range. The coherence between the motor current and vibration signals indicates that rotor eccentricity and bearing faults are reflected in the motor current spectrums. Also the location of bearing fault is balls in the bearing as a result of coherence analysis.

In order to determine precisely which frequency band reflects the bearing fluting damage, the sub-band or the MRA of the faulty signals was performed by dividing them into eight subbands in the frequency range $0-6 \mathrm{kHz}^{(7,12,14)}$. These are given in Table 3 in terms of details $\left(d_{\mathrm{i}}\right)$ and approximations $\left(a_{\mathrm{i}}\right)$.

\begin{tabular}{|ll|lll|}
\hline mations & $(\mathrm{Hz})$ & \multicolumn{3}{|c|}{$(\mathrm{Hz})$} \\
\hline$a 1$ & $0-3000$ & $d 1$ & 3000 & -6000 \\
\hline$a 2$ & $0-1500$ & $d 2$ & 1500 & -3000 \\
\hline$a 3$ & $0-750$ & $d 3$ & 750 & -1500 \\
\hline$a 4$ & $0-375$ & $d 4$ & 375 & -750 \\
\hline$a 5$ & $0-187.5$ & $d 5$ & 187.5 & -375 \\
\hline$a 6$ & $0-93.75$ & $d 6$ & 93.75 & -187.5 \\
\hline$a 7$ & $0-46.875$ & $d 7$ & 46.875 & -93.75 \\
\hline$a 8$ & $0-23.4375$ & $d 8$ & $23.4375-46.875$ \\
\hline
\end{tabular}

MRA implementation is shown in (Fig.9) and (Fig.10). According to these results, 3-6 kHz frequency band, which is named as first detail $\left(d_{1}\right)$ in MRA, is the most dominant band in terms of the similarity. The ratio, which can be calculated between the RMS (root-mean-square) values of vibration measurement and the RMS values of $\left(d_{1}\right)$, increases as the motor bearing degrades toward failure. If looked at other subbands of the vibration measurement, a good trend cannot be seen. For this reason, high frequency vibrations, which take place between $3 \mathrm{kHz}$ and $6 \mathrm{kHz}$, are very affective in bearing fluting. Hence, a feature extraction from considered data could be very effectively realized by using the multi-resolution wavelet analysis technique.

(a)
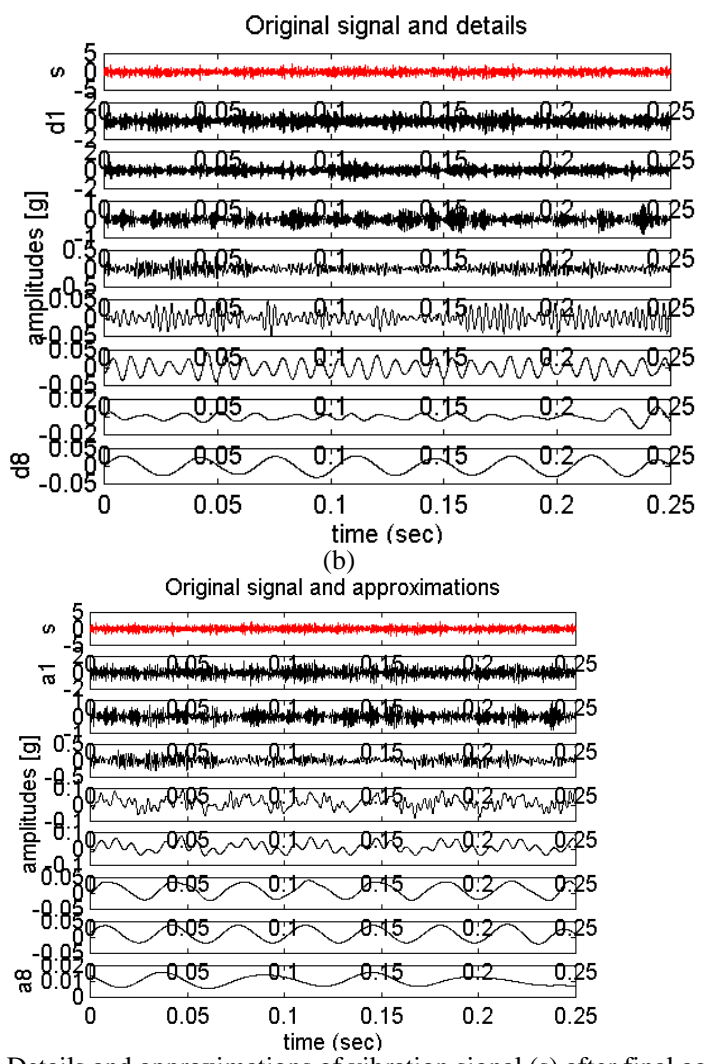

Fig.9. Details and approximations of vibration signal (s) after final aging cycle. (a) Detail sub bands ( $d 1-d 8)$ vibration signal (s) for aged case.

(b) Approximation sub bands ( $a 1-a 8$ ) vibration signal (s) for aged case. 


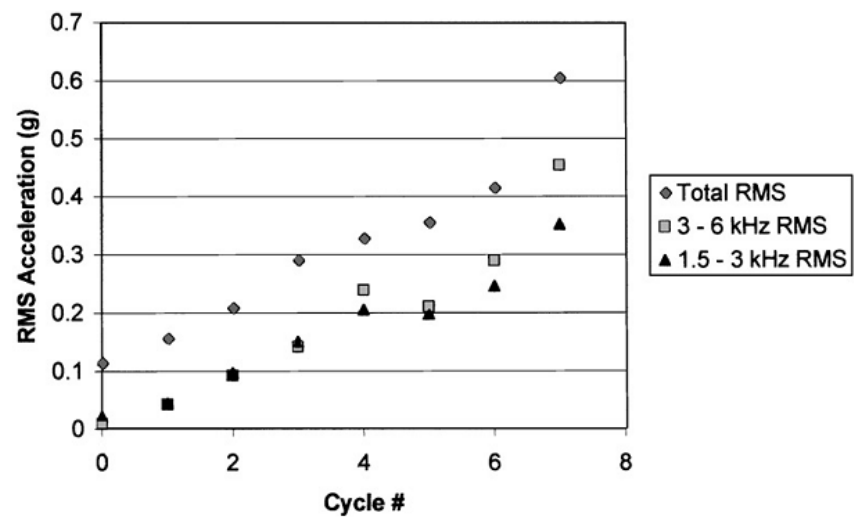

Fig.10. RMS values of vibration signal and two sub-bands (d1 and d2) after final aging cycle.

And also, short-time Fourier transformation (STFT) (Fig.11) of the first detail $\left(d_{1}\right)$ shows that the observed frequency band is between 2 and $4 \mathrm{kHz}$ because of the usage low-pass filter which has a cut-off frequency at $4 \mathrm{kHz}$ during the data acquisition process.

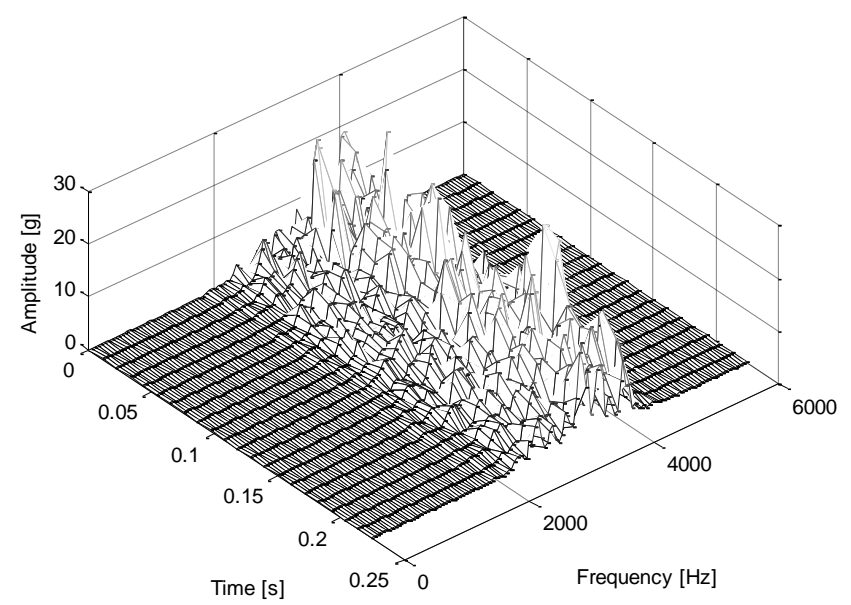

Fig.11. STFT presentation of the sub-band $\left(d_{1}\right)$ related to bearing damage.

The fundamental feature, which is indicated (Fig.11) in the frequency range $2-4 \mathrm{kHz}$, denotes the bearing damage as a result of the bearing fluting test procedures. At this point, we want to ask the question how to detect this before it becomes severe. To answer this, the continuous wavelet transform (Fig.12) is recommended to reveal the potential existence of the bearing damage in early case using only healthy case data.

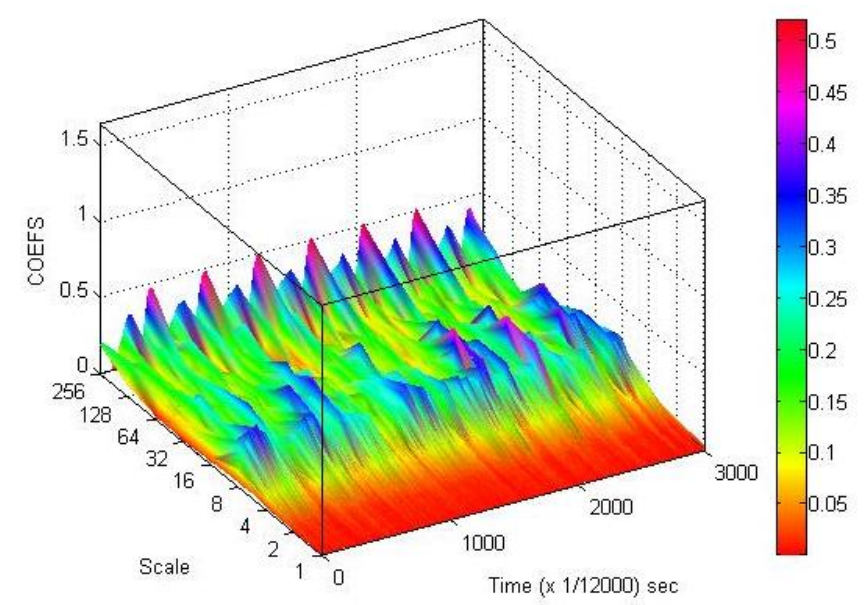

Fig.12. Absolute values of continuos wavelet transform coefficients for scales $1,2,4,8,16,32,64,128,256$ of vibration signals for healthy case.

If the first scale variation is taken outside to plot it individually (Fig.13), it gives the high frequency components which are represented by very small amplitudes, to indicate the origin of the bearing damage as a potential defect.

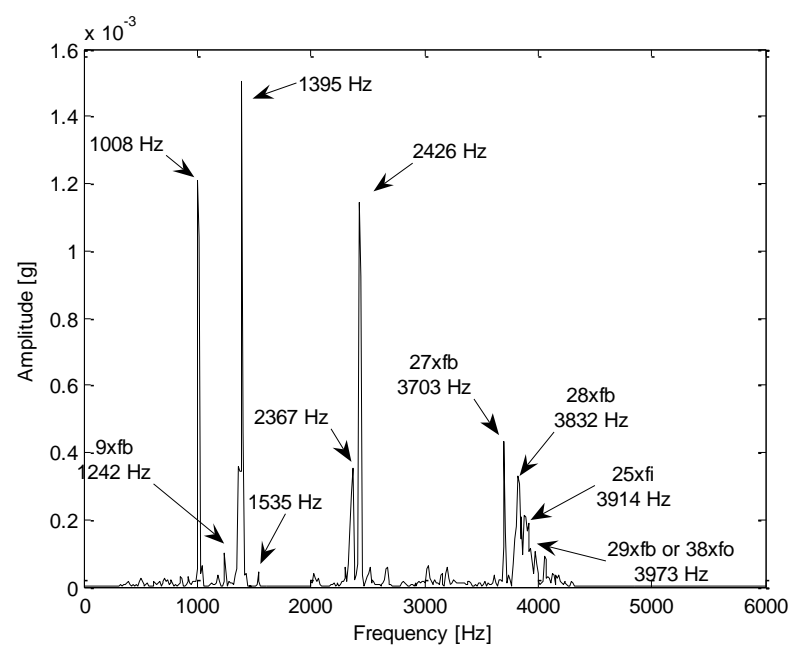

Fig.13. First scale spectrum of vibration signals in healthy case as a potential defect.

AR method is used to model vibration signals parametrically and to determine how the model parameters changes with the aging ${ }^{(15)}$. The sixtieth order model is chosen to construct AR models of vibration signals. The first AR coefficient and variance of white noise input to drive the AR model is increased with the aging (Fig.14 and Fig.15). 


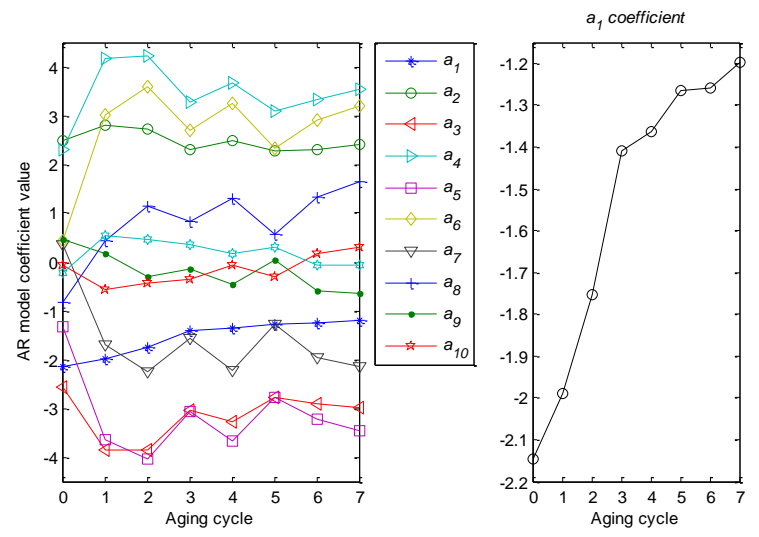

Fig.14. Variation of first $10 \mathrm{AR}$ coefficients (left) and the $a_{1}$ coefficient (right) with aging cycles.

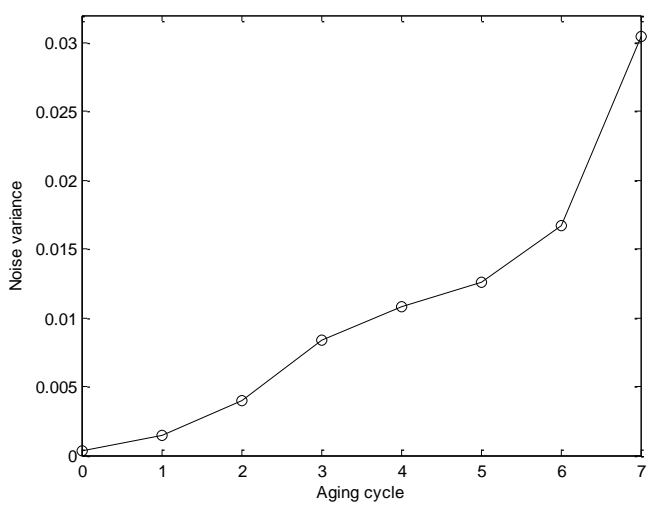

Fig.15. Variance of white noise input to drive the AR models.

Except from vibration and current signals, bearing temperature gives valuable information about the health of the bearings (17) since temperature is an important factor for lubricant. High temperatures reduce the viscosity of lubricant inside of the bearing, and cause early bearing failure. From the relationship between bearing surface temperature and aging (Fig.16), it is seen that the bearing temperature increase with the degradation of the motor. The temperature rise is at most $5^{\circ} \mathrm{C}$ for $\mathrm{M} 8$ by comparing the temperature values of the healthy motors which is cycle \#0 and faulty motors which is cycle \#7. The increase in temperature give information about anomaly, but it is not enough for precise decision on the severity of the fault.

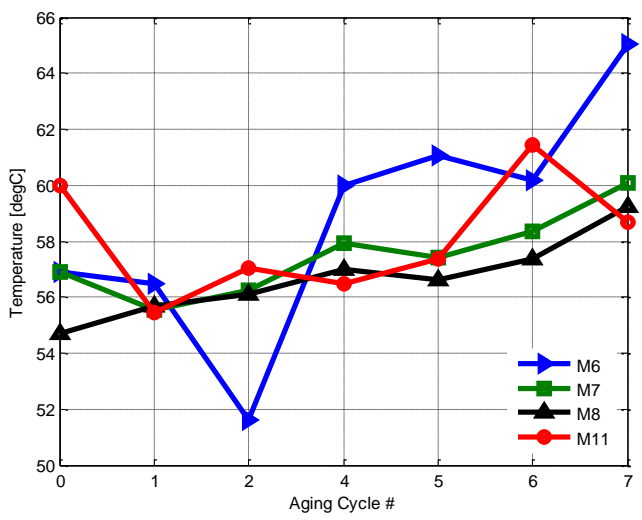

Fig.16. Process-end bearing surface temperature.
ANFIS is used to combine information from several sensors to perform automatic fault detection of bearing failure (Fig.17). The inputs of ANFIS are current, vibration and temperature as given in Table 4, and the output is motor's condition labeled as healthy $(\mathrm{H})$ or faulty $(\mathrm{F})$.

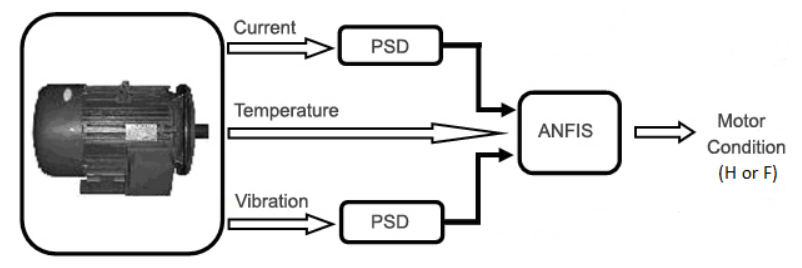

Fig.17. ANFIS based fault detection structure.

Table 4. Inputs used in ANFIS.

\begin{tabular}{|l|c|c|}
\hline \multirow{4}{*}{ Vibration } & Input \# & Frequency band intervals \\
\hline & 1 & $0-732 \mathrm{~Hz}$ \\
\cline { 2 - 3 } & 2 & $2199-2930 \mathrm{~Hz}$ \\
\cline { 2 - 3 } & 3 & $2931-4028 \mathrm{~Hz}$ \\
\hline \multirow{3}{*}{ Current } & 4 & $0-53 \mathrm{~Hz}$ \\
\cline { 2 - 3 } & 5 & $123-234 \mathrm{~Hz}$ \\
\cline { 2 - 3 } & 6 & $240-352 \mathrm{~Hz}$ \\
\hline Temperature & 7 & - \\
\hline
\end{tabular}

The classification performance of ANFIS as healthy or faulty condition is calculated by taking a threshold value of 0.5 . Hence 97.8\% of test data is classified correctly (Fig.18).

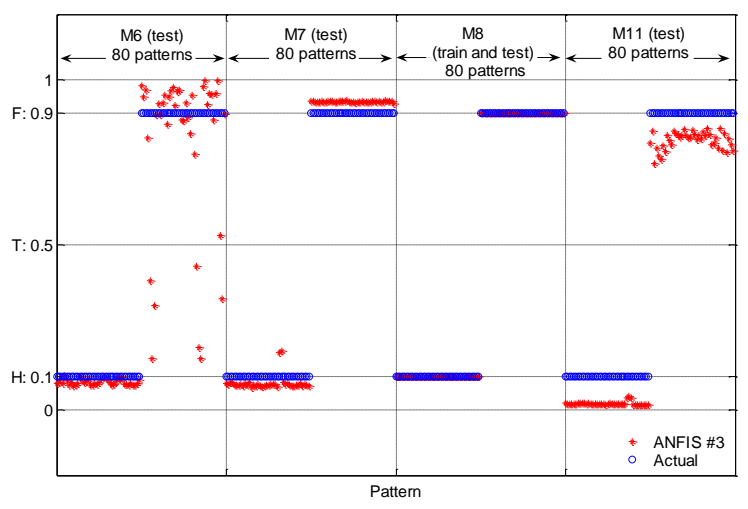

Fig.18. Training and test results for ANFIS. H: Healthy, F: Faulty, T: Threshold

\subsection{ELECTRICAL FAULTS AND THEIR DETECTION}

Stator winding insulation faults are also a common source of failure of electric motors. Especially the use of motor drives creates some undesirable effects with the eventual failure of motors ${ }^{(20)}$. In this section, detection of stator winding insulation fault of main-fed induction motor is addressed.

Stator current imbalances based upon the stator insulation damage act on the stator magneto motor force ( $\mathrm{mmf}$ ) and cause motor vibrations. In this sense the cross spectral approach which is the calculation of coherence function between the motor current and vibration signals for the initial and aged cases (Fig.19) is used to detect the insulation damage. 


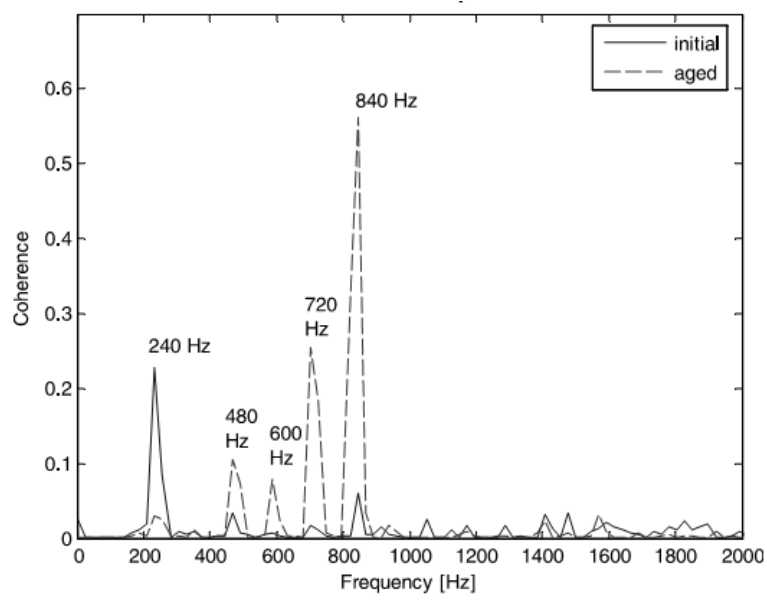

Fig.19. Coherence variation between motor current and vibration signal.

By comparing the initial and aged cases (Fig.19), the even harmonics of line frequency between $4^{\text {th }}$ and $16^{\text {th }}$ in the range $0-1000 \mathrm{~Hz}$, namely the frequencies $240,360,480,600,720$, 840 , and $960 \mathrm{~Hz}$ appear due to insulation aging.

A neuro-detector is designed for the automatic fault detection using the coherence approach to determine the even harmonic effects, which characterize the stator insulation damage. The artificial neural network topology has an auto-associative structure, which uses the same input-output pairs in the frequency domain. Each input-output pairs obtained by coherence approach cover the frequency band of $0-1000 \mathrm{~Hz}$, which contains the most effective even harmonics, and they are defined as training patterns of the training data set of the neural structure. The feed forward neural structure has 50 nodes in terms of the input and output nodes, while number of the hidden nodes is 10. At the first step, the neural network is trained for the normal condition that is healthy stator insulation case using a learning algorithm. After that, the aged case is asked to it as an unknown case. In this case, the neural network produces different responses at the output nodes with a big error changes (Fig.20). Even harmonic values related to the insulation degradation can be detected looking through the error change.

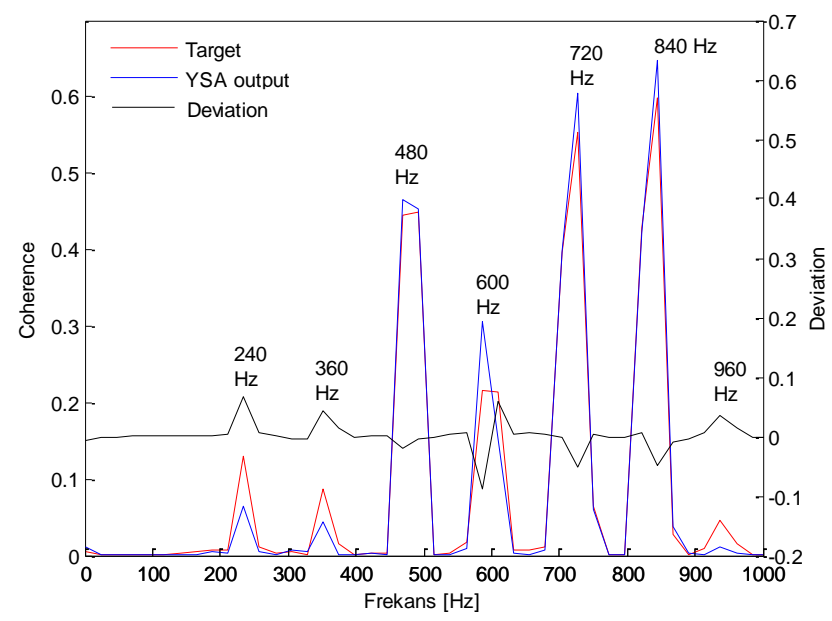

Fig.20. Testing result of the neural network for the aged case.

\section{CONCLUSIONS AND DISCUSSIONS}

In this paper fault detection and diagnosis in induction motors using mathematical methods are discussed with theory and example applications. Eccentricity detection, bearing fault detection and stator winding insulation faults are considered.

Eccentricity is detected by spectrum of stator current and coherence between current and vibration signals.

The statistical analysis of vibration signals give information about the anomaly. Power spectral analysis of vibration signal give the features of bearing fault as an increase of energy in the high frequency region of spectrum The most dominant frequency band is determined by multi-resolution wavelet analysis of vibration signals. Potential bearing fault is detected using continuous wavelet transform. Coherence calculation revealed the location of fault in bearing. AR modeling give the first coefficient of model can be used as a feature of bearing fault. ANFIS application helps to make decision about the condition of motor using spectral features of current and vibration signals, and bearing temperature.

Features for stator winding insulation fault is determined as even harmonics between $4^{\text {th }}$ and $16^{\text {th }}$ in coherence function. A neuro-detector can be used to detect the changes of these harmonics.

\section{REFERENCES}

[1] S.Nandi, H.Toliyat, XLi, (2005). Condition monitoring and fault diagnosis of electrical motors - a review. IEEE Transactions on Energy Conversion. 20 (4), pp.719-729.

[2] A.K.S.Jardine, D.Lin, D.Banjevic, (2006). A review on machinery diagnostics and prognostics implementing condition-based maintenance. Mechanical Systems and Signal Processing. (20), pp.1483-1510.

[3] A.H.Bonnett, (2000). Root Cause AC Motor Failure Analysis with a Focus on Shaft Failures. IEEE Transactions on Industry Applications. 36 (5), pp.1435-1448.

[4] R.R.Schoen, T.G.Habetler, F.Kamran, R.G.Bartheld, (1995). Motor Bearing Damage Detection Using Stator Current Monitoring. IEEE Transactions on Industry Applications. 31 (6), pp.1274-1279.

[5] IEEE Standard Test Procedure for Evaluation of Systems of Insulation Materials for Random-wound AC Electric Machinery, IEEE Std 1171974.

[6] A.S.Erbay, B.R.Upadhyaya, (1999) Multi sensor fusion for induction motor aging analysis and fault diagnosis. Research Report, UTK,UTNE/BRU/99-01. Knoxville, TN, USA.

[7] S.Şeker, E.Ayaz, B.R.Upadhyaya, A.S.Erbay, Analysis of Motor Current and Vibration Signals for Detecting Bearing Damage in Electric Motors. Maintenace And Reliability Conference. Knoxville, TN, USA, 8-10 May 2000, (1) pp.29.01-29.14.

[8] S.Seker, (2000). Determination of Air-Gap Eccentricity in Electric Motors Using Coherence Analysis. IEEE Power Engineering Review. July, pp.48-50.

[9] S.Şeker, E.Ayaz, E.Türkcan, (2003). Elman's Recurrent Neural Network Applications to Condition Monitoring in Nuclear Power Plant and Rotating Machinery. Engineering Applications of Artificial Intelligence. 16 (7-8), pp.647-656.

[10] S.Şeker, E.Ayaz, (2003). A Reliability Model for Induction Motor Ball Bearing Degradation. Electric Power Components \& Systems. 31 (7), pp.639-652.

[11] S.Şeker, E.Ayaz, (2002). A Study on Condition Monitoring for Induction Motors Under the Accelerated Aging Processes. IEEE Power Engineering Review. 22 (7), pp.35-37.

[12] S.Şeker, E.Ayaz, (2003). Feature extraction related to bearing damage in electric motors by wavelet analysis. Journal of the Franklin Institute. 340 (2), pp.125-134. 
[13] E.Ayaz, S.Şeker, E.Türkcan, B.Barutçu, Combination Of Spectral And Multi-Resolution Wavelet Analysis For Fault Detection In Electric Motors. ELECO'2003, Third International Conference on Electrical and Electronics Engineering. Bursa, Turkey, 3-7 December 2003, pp.94-98.

[14] E.Ayaz, A.Öztürk, S.Şeker, B.R.Upadhyaya, (2009). Fault detection based on continuous wavelet transform and sensor fusion in electric motors. COMPEL: The International Journal for Computation and Mathematics in Electrical and Electronic Engineering. 28 (2), pp.454470.

[15] E.Ayaz, (2014). Autoregressive modeling approach of vibration data for bearing fault diagnosis in electric motors. Journal of Vibroengineering. 16 (5), pp.2130-2138.

[16] C.C.Wang, Y.Kang, P.C.Shen, Y.P.Chang, Y.L.Chung, (2010). Applications of fault diagnosis in rotating machinery by using time series analysis with neural network. Expert Systems with Applications. (37), pp.1696-1702.

[17] M.S.Yilmaz, E.Ayaz, Adaptive Neuro-Fuzzy Inference System for Bearing Fault Detection in Induction Motors Using Temperature, Current, Vibation Data. EUROCON 2009-International IEEE Conference. Saint-Petersburg, Russia, May 18-23, 2009.

[18] N.T.Nguyen, H.H.Lee, Bearing fault diagnosis using adaptive network based fuzzy inference system. International Symposium on Electrical \& Electronics Engineering. Vietnam, 24- 25 October 2007.

[19] M.S.Ballal, Z.J.Khan, H.M.Suryawanshi, R.L.Sonolikar, (2007). Adaptive neural fuzzy inference system for the detection of inter-turn insulation and bearing wear faults in induction motor. IEEE Transactions on Industrial Electronics. 54 (1).

[20] E.Ayaz, M.Uçar, S.Şeker, B.R.Upadhyaya, (2009). Neuro-detector based on coherence analysis for stator insulation in electric motors. Electric Power Components and Systems. 37 (5), pp.533-546.

[21] V.S.Vaseghi, (1996) Advanced signal processing and digital noise reduction, New York: John Wiley.

[22] T.K.Moon, W.C.Stirling, (1999) Mathematical Methods and Algorithms for Signal Processing, Prentice Hall.

[23] S.Qian, D.Chen, (1996) The joint Time -Frequency Analysis-Methods and Applications. Englewood Cliffs, NJ: Prentice-Hall.

[24] I. Daubechies, (1990). The wavelet transform, time-frequency localization and signal analysis. IEEE Transactions on Information Theory 36 (5) pp.961-1005.

[25] A.Papoulis, (1987) Probability, Random Variables and Stochastic Processes. McGraw Hill International Edition, $5^{\text {th }}$ printing, Singapore.

[26] M.H.Hayes, (1996) Statistical Digital Signal Processing and Modeling. John Wiley\&Sons, Inc.

[27] L.H.Tsoukalas, R.E.Uhrig, (1997) Fuzzy and neural approaches in engineering. New York: Wiley.

[28] J.S.R.Jang, (1993). Adaptive-network-based fuzzy inference system. IEEE Transactions on Systems, Man, and Cybernetics 23 (3).

\section{ACKNOWLEDGMENT}

The author gratefully thanks to Prof. Serhat Seker from the Istanbul Technical University and Prof. Belle R. Upadhyaya from the University of Tennessee, Knoxville for their support.

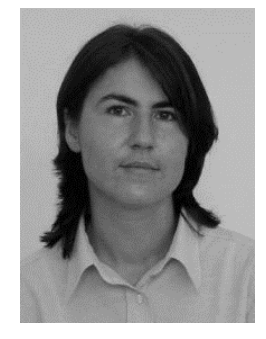

\section{BIOGRAPHIES}

EMINE AYAZ received the BS, MS and PhD. degrees from the Istanbul Technical University (ITU), Electrical Engineering Department, in 1993, 1997 and 2002 respectively. In 1999, she joined to the University of Tennessee, Nuclear Engineering Department and Maintenance \& Reliability Centre to do research on accelerated aging studies of the electric motors. She is currently associate professor in Electrical Engineering Department of ITU. Her research interests are signal processing, soft computing, and condition monitoring in electric power systems. 